\title{
Recent English financial terms in French- language, Dutch-language and German-language financial publications: borrowing versus translating
}

Diana Phillips

\section{(2) OpenEdition}

\section{Journals}

Electronic version

URL: http://journals.openedition.org/asp/3741

DOI: $10.4000 /$ asp.3741

ISSN: 2108-6354

\section{Publisher}

Groupe d'étude et de recherche en anglais de spécialité

\section{Printed version}

Date of publication: 1 December 1995

Number of pages: 81-89

ISSN: 1246-8185

\section{Electronic reference}

Diana Phillips, « Recent English financial terms in French-language, Dutch-language and Germanlanguage financial publications: borrowing versus translating », ASp [Online], 7-10 | 1995, Online since 30 July 2013, connection on 21 April 2019. URL : http://journals.openedition.org/asp/3741 ; DOI $10.4000 / a s p .3741$

This text was automatically generated on 21 April 2019.

Tous droits réservés 


\section{Recent English financial terms in}

French-language, Dutch-language and German-language financial publications: borrowing versus translating

Diana Phillips

\section{EDITOR'S NOTE}

L'auteur de cette contribution n'a pas autorisé sa publication en ligne.

\section{ABSTRACTS}

When using financial terminology, the Dutch language tends to borrow from English much more often than it translates; the same applies to the German language, though to a lesser extent; with the French language, every effort is made to translate.

En matière de terminologie financière, la langue flamande emprunte à l'anglais bien davantage qu'elle ne traduit; l'allemand fait de même à un moindre degré; le français s'efforce, relativement, de traduire. 
INDEX

Mots-clés: emprunt lexical, finance, terminologie

Keywords: lexical borrowing, terminology

\section{AUTHOR}

DIANA PHILLIPS

Diana Phillips teaches at the University of Antwerp-RUCA. diana.phillips@ua.ac.be 